\title{
Noisy Wavefront Propagation in the Fisher-Kolmogorov-Petrovsky-Piscounov Equation
}

\author{
Charles R. Doering ${ }^{* \dagger}$, Carl Mueller** and Peter Smereka* \\ ${ }^{*}$ Department of Mathematics, University of Michigan, Ann Arbor, MI 48109-1109 \\ ${ }^{\dagger}$ Michigan Center for Theoretical Physics, University of Michigan, Ann Arbor, MI 48109-1120 \\ ${ }^{* *}$ Department of Mathematics, University of Rochester, Rochester, NY 14627
}

\begin{abstract}
We discuss some conjectures and open questions regarding the velocity of front propagation in the stochastic Fisher-Kolmogorov-Petrovsky-Piscunov equation
\end{abstract}

\section{INTRODUCTION}

The Fisher-Kolmogorov-Petrovsky-Piscunov (FKPP) equation $[1,2]$ is one of the most fundamental models in mathematical biology and ecology [3]. It describes a population $u(x, t)$ that evolves under the combined effects of spatial diffusion and local logistic growth and saturation. In one space dimension the FKPP equation may be written

$$
\partial_{t} u=D \partial_{x x} u+\gamma u(1-u)
$$

with diffusion coefficient $D$, low-density growth rate $\gamma$, and where the population has been normalized so that the stable saturation level is $u=1$. A particularly interesting aspect of this nonlinear partial differential equation is that it gives a simple description of the invasion of the stable saturated state $(u=1)$ into regions of space occupied by the unstable "extinct" state $(u=0)$. It turns out that after transients, this invasion proceeds via propagation of a front at a constant velocity.

To find these travelling wave fronts we look for solutions of the form

$$
u(x, t)=f(x-c t)
$$

where the speed $c$ is to be determined. Inserting this into the FKPP equation (1) yields an ordinary differential equation for the front shape $f(z)$ :

$$
D f^{\prime \prime}+c f^{\prime}+\gamma f(1-f)=0 .
$$

The boundary conditions for the front shape function are determined by the setup for $u$. If we consider the setup for a right-moving front where $u \rightarrow 1$ as $x \rightarrow-\infty$ and $u \rightarrow 0$ as $x \rightarrow+\infty$, then the conditions for $f$ are $f \rightarrow 1$ as the "time" $z \rightarrow-\infty$ and $f \rightarrow 0$ as $z \rightarrow+\infty$. In this case we look for positive velocities, $c>0$, and the front is identified as the heteroclinic orbit connecting the unstable fixed point $\left(f, f^{\prime}\right)=(1,0)$ and the stable 
equilibrium state $\left(f, f^{\prime}\right)=(0,0)$ of the two-dimensional dynamical system defined by (3). Because $0 \leq u(x, t) \leq 1$ for any initial condition $0 \leq u(x, 0) \leq 1$, we require that $0 \leq f(z) \leq 1$. Then it is easy to see that the determining factor in the analysis is the need to prevent oscillations around 0 near $f=0$. This means that the "friction coefficient" $c$ in the dynamical system for $f$ must be sufficiently high that the stable fixed point $\left(f, f^{\prime}\right)=(0,0)$ is a node and not a spiral. Hence we find acceptable solutions for any speed $c \geq 2 \sqrt{D \gamma}$.

It turns out that for sufficiently sharp initial fronts in $u(x, 0)$, the minimum speed $c_{\min }=2 \sqrt{D \gamma}$ is selected $[1,2]$, albeit very slowly $[4,5]$. That is, for sharp initial fronts any reasonable definition of the instantaneous velocity $v(t)$ satisfies $v(t)=c_{\min }+$ $\mathcal{O}\left(t^{-1}\right)$. This algebraic relaxation of the speed, combined with the fact that the speed is ultimately selected by the properties of the system far ahead of the bulk of the population front, leads to the terminology that the FKPP equation has a pulled front with weak velocity selection. The situation here is to be contrasted with reaction-diffusion fronts connecting metastable states. The velocity of such a pushed front is determined by the nonlinear dynamics in transition region defining the front and the speed adjusts itself exponentially fast, termed strong velocity selection.

Upon reflection it is clear that the extreme sensitivity of the pulled front to the details of the process near the unstable state cannot be robust or, really, physical. Indeed, any modification of the dynamics at the lowest population level where $u \approx 0$-for example due to population discreteness effects-would invalidate the velocity selection mechanism described above.

To fix ideas, consider the spatially homogeneous chemical reaction scheme

$$
A+B \rightarrow A+A \text { at rate } k_{1} \text { and } A+B \rightarrow B+B \text { at rate } k_{2},
$$

and let $N_{A}(t)$ and $N_{B}(t)$ be the number of $A$ and $B$ particles in the system. The reaction conserves the total number of particles so $N=N_{A}+N_{B}$ is a constant of the dynamics. Neglecting discreteness and fluctuation effects altogether leads to a simple description of the kinetics for the fraction of $A$ particles, $U(t)=N_{A}(t) / N$, the logistic equation

$$
\frac{d U}{d t}=\gamma U(1-U)
$$

with $\gamma=k_{1}-k_{2}>0$ (without loss of generality). This level of modeling predicts that any nonzero initial number of $A$ particles will eventually grow to completely dominate the population.

Discreteness and the accompanying "noise" may qualitatively change this picture. If we model the dynamics of (4) by the appropriate Markovian random walk in particle number space [6] and, for convenience here, keep the just leading Markov diffusion process description in the $N \rightarrow \infty$ limit, the deterministic logistic differential equation (5) is replaced by the Itô stochastic differential equation

$$
\frac{d U}{d t}=\gamma U(1-U)+\sigma \sqrt{U(1-U)} \xi(t)
$$

where $\sigma^{2}=\frac{k_{1}+k_{2}}{N}$ and $\xi(t)$ is a Gaussian white noise process with $\langle\xi(t)\rangle=0$ and $\langle\xi(t) \xi(s)\rangle=\delta(t-s)$. The boundary conditions for the state variable $U$ are absorbing at both extremes, $U=0$ and $U=1$, as is obvious from the reaction scheme in (4). 
It is straightforward to compute the probability that an initial population of $A$ particles will eventually become extinct - an eventuality that is simply impossible in the absence of noise. If $U(t)$ starts from (nonrandom) $U_{0}$, this extinction probability is

$$
\operatorname{Prob}\left(U(t) \rightarrow 0 \text { as } t \rightarrow \infty \mid U(0)=U_{0}\right)=\frac{e^{2 \gamma\left(1-U_{0}\right) / \sigma^{2}}-1}{e^{2 \gamma / \sigma^{2}}-1}
$$

which is strictly positive if the initial population contains any $B$ particles at all (i.e., if $\left.0 \leq U_{0}<1\right)$. There is a significant probability of extinction at low population: the probability of extinction is $1-\mathcal{O}\left(U_{0}\right)$ as $U_{0} \rightarrow 0$. Hence we would expect low population levels - such as those in the leading edge of a traveling wavefront in the FKPP equation-to naturally extinguish themselves. It is thus apparent that no mechanism which relies on the exponentially small population out in front of the wave should be physically relevant in the dynamics.

This lack of "structural stability" of the pulled front has been explored in quite alot of detail recently. In 1997 and 1998 Brunet and Derrida [7] and Kessler, Ner and Sander [8] considered the influence of discreteness in the population variable $u$ on the front speed by replacing the $u(1-u)$ growth and saturation dynamics in the FKPP equation with $u(1-u) \Theta\left(u-N^{-1}\right)$, where $\Theta(\zeta)$ is the step function vanishing when $\zeta<0$. Here $N^{-1} \ll 1$ represents the discreteness level. Repeating the dynamical systems argument for the velocity of a traveling wavefront, they concluded that the selected speed is unique, strongly selected, and given for large $N$ by

$$
c \sim \sqrt{D \gamma}\left[2-\frac{\pi^{2}}{(\log N)^{2}}\right]=c_{m i n}-\mathcal{O}\left((\log N)^{-2}\right) .
$$

This is slower than the minimum speed previously available. Moreover, it displays extremely slow convergence to the "continuum" limit for $u$ as $N \rightarrow \infty$. Soon thereafter Pechenik and Levine [9] computationally verified this kind of velocity dependence on $N$ in another stochastic model. Most recently Brunet and Derrida conjectured as well that in a discrete stochastic model the wavefront should diffuse with an $\mathcal{O}\left((\log N)^{-3}\right)$ diffusion coefficient [10]. See also the contribution of Panja to this volume [11].

\section{THE WAVEFRONT IN THE STOCHASTIC FKPP EQUATION}

In view of the considerations above, we are led to study the stochastic FisherKolmogorov-Petrovsky-Piscounov (sFKPP) equation

$$
\partial_{t} u=D \partial_{x x} u+\gamma u(1-u)+\epsilon \sqrt{u(1-u)} \eta(x, t)
$$

where $\eta(x, t)$ is a gaussian white noise process in space and time satisfying $\langle\eta(x, t)\rangle=0$ and $\langle\eta(x, t) \eta(y, s)\rangle=\delta(x-y) \delta(t-s)$. This stochastic partial differential equation with multiplicative noise is to be interpreted as the continuum limit of a spatially discretized set of Itô equations; more on this below. We will refer to the coefficient $\epsilon$ as the noise strength, which one may think of as being proportional to $1 / \sqrt{N}$ where $N$ is 


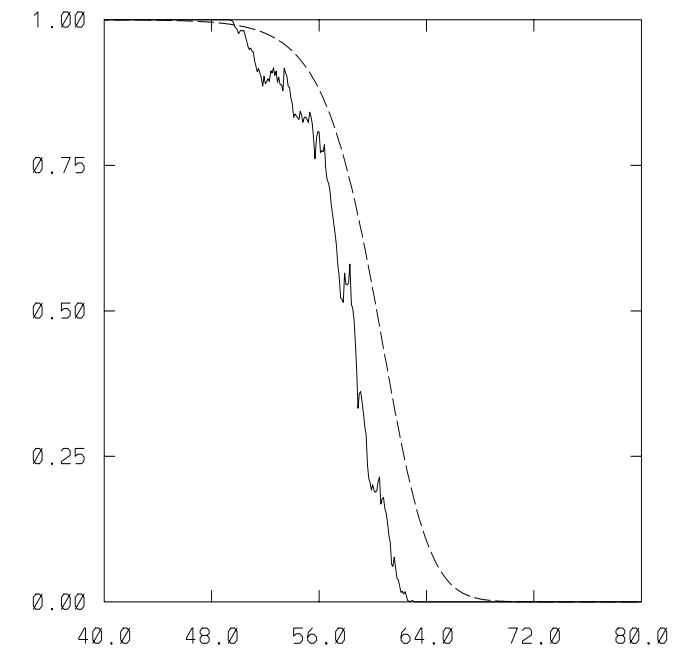

FIGURE 1. Snapshot of a traveling wave for the FKPP (dashed line) and the stochastic sFKPP equation (solid line). The results illustrate that the stochastic wavefront has compact support.

the saturation population at a lattice site or in an appropriately defined correlation volume. There have been many studies of the effects of spatial inhomogeneities and environmental randomness of the FKPP front dynamics, too [14], but we focus on this (statistically) spatially homogeneous model.

Although it is tempting to identify the sFKPP equation (9) as the fluctuating hydrodynamic description of a spatially extended reaction-diffusion model of (4), the extra noise introduced by the microscopic diffusive hopping complicates that connection. The sFKPP has, however, been rigorously derived as just such a description of an appropriate hydrodynamic limit of the contact process with long-range interactions [12].

The multiplicative noise in the sFKPP has profound qualitative effects on the front propagation. In 1995 Mueller and Sowers [13] proved that the sFKPP equation (9) has the so-called compact support property. This means, for example, that if the initial data $u(x, 0)$ for the stochastic partial differential equation satisfies $u(x, 0)=1$ for $x \leq a$ and $u(x, 0)=0$ for $x \geq b$ where $-\infty<a<b<\infty$, then for all subsequent $t>0$ the solution will satisfy $u(x, t)=1$ for $x \leq a(t)$ and $u(x, t)=0$ for $x \geq b(t)$ for some values $a(t)$ and $b(t)$ satisfying $-\infty<a(t)<b(t)<\infty$. That is, a front with sharp leading and trailing edges will always have sharp leading and trailing edges. This is despite the tendency for the diffusion to propagate $u$ into the vacuous regions in front of the wave, and to decrease the population from the saturation level at the back of the front. The noise overwhelms the diffusive transport near the boundaries of the state variable at $u=0$ and $u=1$.

The compact support property of this process can be seen in numerical simulations. In Figure 1 we plot the results of direct numerical simulations of the FKPP equation (1) and stochastic sFKPP equation (9) explicitly showing the qualitative difference in the leading and trailing edges. 


\section{DUALITY AND INTERACTING PARTICLE SYSTEMS}

The FKPP equation (1) is the mean-field hydrodynamic description of many microscopic reaction-diffusion systems including the single-species growth-coagulation process

$$
A \rightarrow A+A \text { at rate } \gamma \text { and } A+A \rightarrow A \text { at rate } \chi,
$$

where $u$ would be a measure of the local density of $A$ particles normalized by the equilibrium density $\sim \gamma / \chi$. It is tempting to interpret the stochastic sFKPP equation as a fluctuating hydrodynamic description of this process, as well, but a moment's reflection shows that this cannot be. Indeed, fluctuations around the equilibrium level of (10) corresponding to $u=1$ do not vanish for the $A \rightleftharpoons A+A$ process as they do (by definition) in the sFKPP equation. For (10) one would expect instead that the fluctuation strength should be proportional to at least $\sqrt{u}$ up to and beyond the $u=1$ level.

Nevertheless there is an exact rigorous relationship between the process in (10) and the stochastic Fisher-Kolmogorov-Petrovsky-Piscounov equation (9). The connection is by way of the notion of duality, an idea from the branch of probability theory concerned with interacting particle systems [15]. The duality relation between the growthcoagulation reaction and the sFKPP equation was discovered in 1986 by Shiga and Uchiyama [16]. It is most easily described in the discrete space setting; see [17] for a complete - and, we hope, accessible — derivation of this duality relation.

Impose a lattice on the $x$-axis with lattice spacing $h$ and consider the discretized sFKPP equation

$$
d U_{i}(t)=\left[\frac{D}{h^{2}}\left(U_{i+1}-2 U_{i}+U_{i-1}\right)+\gamma U_{i}\left(1-U_{i}\right)\right] d t+\sigma \sqrt{U_{i}\left(1-U_{i}\right)} d W_{i}
$$

where the $W_{i}(t)$ are independent Brownian motions at each lattice site. The continuum limit of this system - taking $h \rightarrow 0$ with $x=i h$-is exactly the sFKPP equation (9) when we also send $\sigma \rightarrow \infty$ so that $\epsilon^{2}=\sigma^{2} h=\mathcal{O}(1)$.

On the other hand consider the spatially extended $A \rightleftharpoons A+A$ process (10) on the same spatial lattice where particles at site $i$ hop to neighboring sites $i \pm 1$ at rate $\frac{D}{h^{2}}$ independently of all other processes. The rate coefficients $\gamma$ and $\chi$ of the Markov process for the system are defined precisely as follows: If there are $n_{i}$ particles at site $i$ at time $t$, then they produce another particle there $\left(n_{i} \rightarrow n_{i}+1\right)$ at rate $n_{i} \gamma$, and the pair coagulation process $\left(n_{i} \rightarrow n_{i}-1\right)$ proceeds at rate $\frac{1}{2} n_{i}\left(n_{i}-1\right) \chi$. It is straightforward to write down the master equation for the joint probability distribution of all the occupation numbers $\left\{N_{i}(t)\right\}$ at all the lattice sites.

Duality between these systems holds when the noise amplitude $\sigma^{2}$ equals the coagulation rate $\chi$. It is a connection between one processes evolving forward in time and the other evolving backwards in time: let $0 \leq t \leq T$, then $\sigma^{2}=\chi \Rightarrow$

$$
\left\langle\prod_{i}\left(1-U_{i}(t)\right)^{N_{i}(T-t)}\right\rangle=\left\langle\prod_{i}\left(1-U_{i}(T-t)\right)^{N_{i}(t)}\right\rangle
$$

where the expectation $\langle\cdot\rangle$ is over both (independent) processes $\left\{U_{i}(t)\right\}$ and $\left\{N_{i}(t)\right\}$. 
The duality relation allows us to express moments of the solution of the (discrete) sFKPP equation (11) at time $t$ in terms of its initial data and the solution of the reactiondiffusion system. In particular, if we choose the initial condition for the particles so that there is just one particle at site $i$ and no others anywhere else (i.e., $N_{j}(0)=\delta_{i j}$ ), then

$$
\left\langle U_{i}(t)\right\rangle=1-\left\langle\prod_{j}\left(1-U_{j}(0)\right)^{N_{j}(t)}\right\rangle .
$$

And if the initial data for $\left\{U_{j}\right\}$ is $U_{j}(0)=1$ if $j \leq 0$ and $U_{j}(0)=0$ if $j>0$ (a setup for a wavefront propagating to the right), then duality produces the representation

$\left\langle U_{i}(t)\right\rangle=\operatorname{Prob}($ a site $j \leq 0$ has a particle at time $t \mid$ only one particle at site $i$ at time 0 ).

Note that the growth-coagulation process never becomes extinct: if we start with one (or more) particles we are ensured that there will always be at least one particle somewhere on the line. In fact, we expect that starting with only one singly occupied site $i$, the growth process will dominate until the unique equilibrium steady state with average occupation number $2 \gamma / \chi$ at all lattice sites is achieved.

This connection implies that the asymptotic front speed for the sFPKK equation should be the same as the asymptotic front speed for the $A \rightleftharpoons A+A$ reaction-diffusion system - when the coagulation rate $\chi$ is the same as the square of the noise amplitude $\sigma^{2}$. In general we do not know an exact expression for the velocity of a front in the interacting particle model. However, we do know many things about a certain limit of the single-species growth-coagulation reaction-diffusion system, the so-called diffusioncontrolled limit.

The diffusion-controlled $A \rightleftharpoons A+A$ process is the reaction-diffusion dynamics in (10) in the limit where $\chi \rightarrow \infty$ so that $A+A \rightarrow A$ instantaneously when two particles land on the same lattice site [18]. The equilibrium density $\rho_{e q}$ of particles on the lattice is then nonzero only if the growth rate $\gamma$ is also scaled up appropriately as $\chi \rightarrow \infty$. (By "density" we mean that $\rho_{e q} h$ is the equilibrium occupation probablility - equivalently the average occupation number - of a site.) Noting the correspondence of $\chi$ and $\sigma^{2}$ in the duality relation, this means that the strong-noise limit of the sFKPP equation is dual to the diffusion-controlled limit of the interacting particle process. We can make a quantitative connection between the system near the limit and the diffusion-controlled limit itself if we identify the equilibrium occupation probabilities $\rho_{e q} h$ and $2 \gamma / \chi=2 \gamma / \sigma^{2}$.

The front propagation problem for the diffusion-controlled single-species growthcoagulation reaction-diffusion system has been solved exactly and in great detail [18, 19, 20]. In particular we know that the speed of the wavefront for the diffusion-limited process is $c=D \rho_{e q}[19,20]$. From this and the duality connection we can conjecture the behavior of the velocity of the sFKPP wave in the strong noise limit:

$$
c=D \rho_{e q} \sim D \frac{2 \gamma}{h \sigma^{2}}=\frac{2 D \gamma}{\epsilon^{2}} .
$$

In Figure 2 we plot the front velocities normalized by the minimum no-noise speed $(2 \sqrt{D \gamma})$ as measured in direct numerical simulations of the (discrete) sFKPP equation (11) versus the dimensionless noise strength $\epsilon /(D \gamma)^{\frac{1}{4}}$. The solid lines are the asymptotic conjectures (8) for weak noise (using $N=2 \gamma / \sigma^{2}$ ) and (14) for strong noise. 


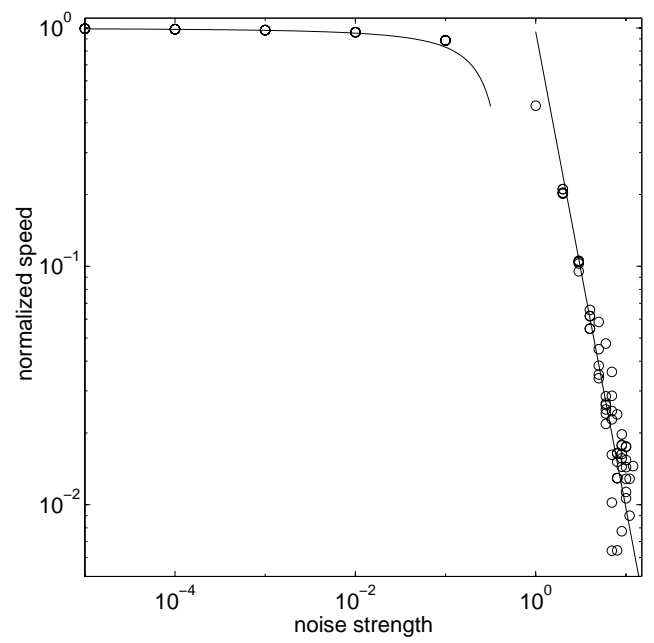

FIGURE 2. Wave speed (normalized by the no-noise speed) vs. dimensionless noise strength from the simulations (data), and the asymptotic conjectures for weak and strong noise (lines).

\section{CONCLUSIONS AND UNSOLVED PROBLEMS}

It is clear from Figure 2 that both the weak and strong noise conjectures for the front propagation speed are quantitatively accurate. Not unexpectedly, some fundamental equations remain:

1. Can these weak and strong noise asymptotic expressions for the front velocity be proven by analysis of the stochastic partial differential equation (with or without the use of duality)?

2. What can we discover about the statistics of the leading and trailing points of the front? (Mueller and Sowers proved that the fluctuating width of the front attains a stationary distribution as $t \rightarrow \infty$ [13]. What about front diffusion?)

3. In the other direction, what does the stochastic partial differential equation tell us about the statistical mechanics of the $A \rightleftharpoons A+A$ growth-coagulation reactiondiffusion process?

4. What can we deduce about front motion in higher spatial dimensions?

This last question in particular is of great interest for a variety of growth processes. In higher spatial dimensions even an initially smooth front can develop structure in the transverse direction(s) and fluctuations may play an even more dramatic role in the dynamics. Much recent effort has gone into studying particle systems whose mean-field descriptions are the higher dimensional FKPP equation [21, 22, 23, 24]. In these systems the effects of discreteness, fluctuations and noise may be very subtle and the quantitative behavior is difficult to discern. 


\section{ACKNOWLEDGMENTS}

CRD thanks Profs. Lutz Shimansky-Geier (Humboldt University) and Peter Jung (Ohio University) for thoughtful comments. We acknowledge research support from NSF and NSA.

\section{REFERENCES}

1. R. A. FISHER, The wave of advance of advantageous genes, Annals of Eugenics 7, 353 (1937).

2. A. Kolmogorov, I. Petrovsky, And N. Piscounov, Etude de l'equation de la diffusion avec croissance de la quantité de matière et son application a un probleme biologique, Moscow University Bulletin of Mathematics 1, 1 (1937).

3. J. A. MurRaY, Mathematical Biology (Springer-Verlag, Berlin, 1998).

4. M. BRAMSON, Convergence of Solutions of the Kolmogorov Equations to Traveling waves, Memoirs of the American Mathematical Society 44 (1983).

5. U. EBERT AND W. VAN SAARLOOS, Front propagation into unstable states: universal algebraic convergence towards uniformly translating pulled fronts, Physica D 146, 1 (2000).

6. N. G. VAN KAMPEN, Stochastic Processes in Physics and Chemistry (North-Holland, Amsterdam, 1981).

7. E. BRUnet AND B. Derrida, Shift in the velocity of a front due to a cutoff, Physical Review E 56, 2597 (1997)

8. D. A. KESSLER, Z. NER, AND L. M. SANDER, Front propagation: Precursors, cutoffs and structural stability, Physical Review E 58, 107 (1998).

9. L. PECHENIK AND H. LEVINE, Interfacial velocity corrections due to multiplicative noise, Physical Review E 59, 3893 (1999).

10. E. BRUNET AND B. DERRIDA, Effect of microscopic noise on front propagation, Journal of Statistical Physics 103, 269 (2001).

11. D. PANJA, Surprising Aspects of Fluctuating "Pulled" Fronts, UPoN 2002 (this volume).

12. C. Mueller And R. TRIBE, Stochastic PDEs arising from the long range contact and long range voter processes, Probability Theory \& Related Fields 102, 519 (1995).

13. C. Mueller And R. B. Sowers, Random travelling waves for the KPP equation with noise, Journal of Functional Analysis 128, 439 (1995).

14. J. XIN, Front propagation in heterogeneous media, SIAM Review 42, 161 (2000).

15. T.M. LiggetT, Interacting Particle Systems (Springer-Verlag, Berlin, 1985).

16. T. Shiga AND K. Uchiyama, Stationary states and the stability of the stepping stone model involving mutation and selection, Probability Theory \& Related Fields 73, 87 (1986).

17. C. R. Doering, C. Mueller and P. Smereka, Physica A (submitted, 2002).

18. M. A. BurschKa, C. R. DoERING, AND D. BEN AVRAHAM, Statics and Dynamics of a DiffusionLimited Reaction: Anomalous Kinetics, Nonequilibrium Self-Ordering, and a Dynamic Transition, Journal of Statistical Physics 60, 695 (1990).

19. C. R. Doering, M. A. BurschKa, AND W. Horsthemke, Fluctuations and correlations in a diffusion-reaction systems: Exact hydrodynamics, Journal of Statistical Physics 65, 953 (1991).

20. D. Ben Avraham, Fisher waves in the diffusion-limited coalescence process $A+A \rightleftharpoons A$, Physics Letters A 247, 53 (1998).

21. J. Riordan, C. Doering, And D. Ben-Avraham, Fluctuations and stability of Fisher waves, Physical Review Letters 75, 565 (1995).

22. G. TRIPATHY AND W. VAN SAarloos, Fluctuation and relaxation properties of pulled fronts: A scenario for nonstandard Kardar-Parisi-Zhang scaling, Physical Review Letters 85, 3556 (2000).

23. G. Tripathy, A. Rocco, J. Casademunt, and W. van SaArloos, The universality class of fluctuating pulled fronts, Physical Review Letters 86, 5215 (2001)

24. E. Moro, Internal fluctuations effects on Fisher waves, Physical Review Letters 87, 238303.1-4 (2001) 